\title{
Multiple Hit Readout of a Microchannel Plate Detector With a Three-Layer Delay-Line Anode
}

\author{
Ottmar Jagutzki, Alfred Cerezo, Achim Czasch, Reinhard Dörner, Mirko Hattaß, Min Huang, Volker Mergel,
} Uwe Spillmann, Klaus Ullmann-Pfleger, Thorsten Weber, Horst Schmidt-Böcking, and George D. W. Smith

\begin{abstract}
We have developed a delay-line readout technique for microchannel plate detectors with an increased acceptance for multiple hit events compared to standard two-layer delay-line anodes. This technique allows unambiguous determination of arrival time and position of at least two simultaneously detected particles, and/or to detect an even larger number of particles in a shower, as long as any two particles do not arrive both at the same time and at the same position within certain limits. We demonstrate and discuss the abilities and limitations of this technique and the relevance for certain experimental tasks.
\end{abstract}

Index Terms-Delay line, fragment imaging, imaging, microchannel plate, multihit, three-dimensional (3-D) detection.

\section{INTRODUCTION}

D ETECTION of single particles or photons with a microchannel plate (MCP) is a well-developed technique [1]. Most applications require the determination of position and/or arrival time of quasistatistically arriving particles, i.e., photons from a celestial object or particles from a laboratory source. There are several readout methods either with phosphor screen-charged coupler device (CCD) or single particle detection schemes in use, depending on the specific task [2]-[13].

For some applications, however, there is need to detect a burst of particles, i.e., events of quasisimultaneously arriving particles. Position and time of each particle shall be recorded. We define such a multihit event as a burst of particles (at least two) arriving within a few microseconds, as the fastest electronic readout cycle for single particles is typically one microsecond. An example for an application is the collision-induced breakup

Manuscript received November 21, 2001; revised June 14, 2002. The initial testing and construction of the Hexanode was supported by RoentDek GmbH, Kelkheim, Germany, and by Oxford Nanoscience Limited, U.K., under a DTI SMART award, and by the European Commission through a BRITE/EURAM project (BE4357) under Contract BRPR-CT97-0520.

O. Jagutzki and K. Ullmann-Pfleger are with the Institut für Kernphysik, Universität Frankfurt, and also with RoentDek GmbH, Kelkheim, Germany (e-mail: jagutzki@hsb.uni-frankfurt.de)

A. Cerezo and G. Smith are with Oxford Nanoscience Ltd. and with the Department of Materials, Oxford University, Oxford OX1 3PH, U.K. (e-mail alfred.cerezo@materials.oxford.ac.uk).

A. Czasch, R. Dörner, M. Hattaß, U. Spillmann, T. Weber, and H. SchmidtBöcking are with Institut für Kernphysik, Universität Frankfurt, 60486 Frankfurt, Germany.

M. Huang is on leave from the Department of Materials, Oxford University, Oxford OX1 3PH, U.K. He is now with Screen Technology Ltd., Cambridge, U.K.

V. Mergel is with the Institut für Kernphysik, Universität Frankfurt, and also with RoentDek GmbH, Kelkheim, Germany, on leave from Institut für Kernphysik, Universität Frankfurt, 60486 Frankfurt, Germany.

Digital Object Identifier 10.1109/TNS.2002.803889 of a molecule into several charged fragments and the projection of the fragments onto one microchannel plate detector [14].

A microchannel plate will respond to a large number of almost simultaneously arriving particles (a multihit event) in the same way as to statistically arriving (single) particles, as long as the integrated particle rate over time (multihit rate times average multiplicity) does not exceed the total count rate limit. There is also a local count rate limit depending on pore size and single pore recovery time. Consequently, the standard "image intensifier" technique, i.e., projection of the charge cloud(s) from the MCP stack onto a phosphor screen and recording the optical image on the screen with a CCD camera, will also provide information on the positions of the individual particles in a shower, as it does for singly arriving particles. This requires already an "advanced" CCD readout scheme so that each multihit event is individually recorded. Still, this technique cannot be used to analyze the relative arrival times of the individual particles in a multihit as defined above due to the low frame rate (image readout frequency) of even the fastest CCD cameras.

Several alternative MCP readout concepts exist for precisely detecting the position and time for single particles, but only few of them can analyze multihit events.

From these techniques pixel detectors with a segmented anode and a large number of truly independent and fast electronic channels can be considered to have the highest potential for position and time sensitive multihit detection [15], [16], while methods using slow charge integrating circuits (such as the resistive anode) will not be adequate at all for such tasks.

Pixel techniques and crossed wire anodes suffer from an escalating electronic complexity as the demand on position resolution increases. Therefore, hybrid techniques of CCD readout (precise imaging) and pixel readout (precise timing) have been adopted [17], [18]. Again, the complexity of such special detector set-ups and especially the usually rather low frame rates of the CCD component require the application of alternative concepts.

Here we investigate the method of delay-line readout for position and time sensitive detection of particle showers with microchannel plates.

Several types of delay-line anodes for MCP have been described in literature [8]-[13]. These have in common to contain individual delay chains for each of the two directions in the detection plane. The charge cloud is collected either on comb-like structures where the individual "fingers" are separated by a signal-delaying circuit, or on a meander or spiral shaped track/wire which acts as a collector and delay-line 
simultaneously. From the relative delay that the signals experience along the lines before they arrive at the two ends of the delay line the position along this direction can be determined. The two-dimensional position is then obtained by reading the second (perpendicular) delay-line in coincidence. The time signal pick-up from the MCP finally completes the information on a detected particle (position and time).

The position resolution is much superior than for pixel detectors as a resolution much better than the pitch distance of the collecting wires/tracks is achieved. This is due to an inherent center-of-gravity averaging on the anode. Position resolutions well below $50 \mu \mathrm{m}$ have been reported [11] and can be maintained for large-scale detectors [19]. The time resolution is well below one nanosecond. Only five electronic timing channels are required to obtain this information. As electronic timing circuits are comparably fast (unlike charge integrating circuits) several consecutive signals can principally be handled on the chains with rather low dead time. Due to redundancy and checksums in the signal sequences (as described in the next section) it is even possible to analyze particle pairs that arrive within the electronic dead time as long as they are sufficiently separated in position.

The suitability of the delay-line technique for multihit detection depends mostly on the ability of the electronic chains to determine the timing for several signals following each other on the same line. There is an ambiguity between time differences originating from relative positions on the detector and from the particle arrival time difference on the MCP that must be resolved.

For better resolving of this ambiguity we have developed a three-layer hexagonal delay-line ("Hexanode") [20]. The concept of using additional anode layers has already been applied for similar experimental tasks using gas-filled particle detectors [21]-[24]. Our Hexanode approach for MCP readout follows the design of the "standard" helical wire anode (HDL) as reported in [8]. The HDL can already be used for a variety of multihit detection tasks if combined with adequate electronic readout circuits [25]. In Sections II and III, we will describe this technique and discuss its limitations. In Sections IV and IV we present the three-layer hexagonal design and discuss the advantages for multihit applications. We conclude with prospects for future electronic readout schemes.

\section{Position and Time Sensitive Particle Detection} With a (Standard) Helical WiRE DElay-Line ANODE

The typical HDL [8] consists of two layers of wire pairs, each pair wound around insulating rods at the edges of a rectangular metal base plate. The layers are aligned perpendicular to each other. The two insulating rods have different thickness, so that the inner and outer layers are separated by a gap of typically $1 \mathrm{~mm}$. For each layer the pair of wires forms a transmission line with low dispersion for a differential signal. The distance of the wires is typically $0.5 \mathrm{~mm}$, so the distance per transmission line loop is $1 \mathrm{~mm}$. The wires collect the electrons from an MCP charge cloud. By applying an extraction voltage to the wires of typically $500 \mathrm{~V}$ with respect to the MCP exit over a distance of several millimeters, a proper charge cloud broadening is ensured to allow for a center-of-gravity averaging over the wire loops. If one of the wires (signal wire) from each layer is set to a slightly more positive voltage (typically $50 \mathrm{~V}$ ) than the other (reference wire), the electrons are collected on the signal wire only. The charge cloud is almost equally shared between the signal wire loops of both layers, giving rise to differential signals on the two perpendicular transmission lines, the signal height being proportional to the charge in the cloud. The pulse shape depends on the number of wire loops that have collected a portion of the cloud, folded with the signal delay per loop and the time structure of the charge cloud emerging from the MCP. The signal propagates to the ends of each transmission line with a speed close to speed of light. DC-decoupled differential amplifiers and constant fraction discriminator (CFD) circuits are used to produce four fast (norm) timing signals for each delay line end. The time sequence of these signals codes the position in both dimensions. A fifth amplifier and CFD connected to the MCP (dc-decoupled on bias contact) can be used to determine the time of the particle impact. An outer trigger is then needed to define a (common) time zero for these five arrival time signals. The position of the particles $(X, Y)$ with respect to the center of the detector is given by

$$
\begin{aligned}
& X=\left(x_{1}-x_{2}\right) \cdot v_{\perp} \\
& Y=\left(y_{1}-y_{2}\right) \cdot v_{\perp} .
\end{aligned}
$$

The time-of-flight (TOF) is simply

$$
T O F=t
$$

With $x_{1}, x_{2}, y_{1}, y_{2}$, and $t$ denominating the measured signal arriving times with respect to time zero for the four delay-line ends and the MCP. $v_{\perp}$ is the effective perpendicular propagation speed on the delay-line anode (typically $1 \mathrm{~mm} / \mathrm{ns}$ ). Note that $v_{\perp}$ is much smaller than the real signal speed parallel to the wires. For simplification we assume here that $v_{\perp}$ is the same for both delay-line layers.

For pure imaging application usually only two time digitizers are used that directly measure $x_{1}-x_{2}$ and $y_{1}-y_{2}$, by two independent start/stop clocks, $x_{1}$ being the "start" and $x_{2}$ the "stop" signal (after delay), respectively, for $Y$ dimension.

However, if the absolute time information (e.g., the TOF) is of interest it is advantageous not only to add a third timing channel for the MCP signal but to measure all five timing signals with respect to a common time zero. The redundancy gives rise to the so-called time sum spectrum for $X$ and $Y$ direction. If external delays like varying cable lengths and electronic response times are subtracted it is

$$
T_{\text {sum }}=x_{1}+x_{2}=y_{1}+y_{2}=\text { const. }
$$

$T_{\text {sum }}$ is independent from the particle position and corresponds numerically to the single path delay of the delay line anode (typically $50 \mathrm{~ns}$ ) if the MCP signal would be used as time zero $(t=0)$. This well known constant can for example be used for consistency checks. For a "real" HDL one finds that $T_{\text {sum }}$ shows some variation with position. If corrected for this, the width [full-width at half-maximum (FWHM)] of a time sum spectrum is smaller than 500 ps (see Fig. 1). 


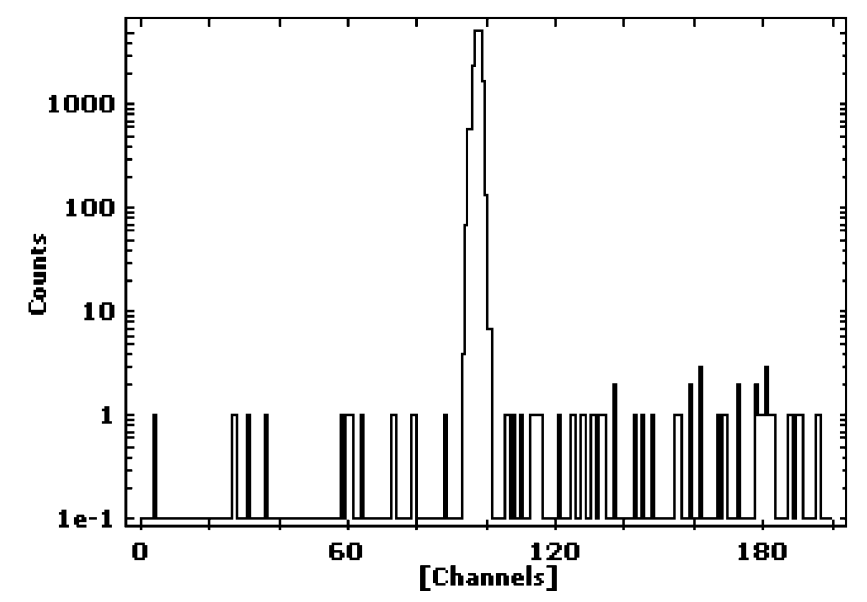

Fig. 1. Time sum spectrum of $T_{\text {sum }}=x_{1}+x_{2}$ from a HDL anode. The bin size is $90 \mathrm{ps}$. Only data within a $5 \mathrm{~mm}$ by $5 \mathrm{~mm}$ position window are displayed here. The FWHM of the peak is 220 ps. The width of this peak is constant over the whole detector (here, 45 -mm diameter), however the absolute peak position can vary by about 1 ns.

Due to the center-of-gravity averaging the achievable position resolution is only determined by the relative time resolution of $x_{1}$ and $x_{2}, y_{1}$ and $y_{2}$, respectively. If $\sigma_{\mathrm{dx}}$ is the time resolution for $X$, one finds for the position resolution

$$
\sigma_{X}=\sigma_{\mathrm{dx}} \cdot v_{\perp}
$$

We assume for simplicity here that $\sigma_{X}=\sigma_{Y}$. Note, that usually $\sigma_{\mathrm{dx}}$ is found to be smaller than $\sigma_{t}$, the absolute time resolution for the TOF.

Especially for analyzing multihit events the time sum can serve for more than just a consistency check, it is an important source of information. First of all, (1) and (2) allow redundant determination of the position and time for a particle. If for some reason one of the signals on the delay-line ends (e.g., $y_{2}$ ) has not been registered, the $Y$ position information can still be recovered:

$$
Y=\left(2 \cdot y_{1}-T_{\mathrm{sum}}\right) \cdot V_{\perp} \text {. }
$$

Equivalent equations hold for $x_{1}, x_{2}$, and $y_{2}$.

If the time zero is defined by an outer trigger, the TOF of the particle with respect to that trigger can be determined without the MCP signal $t$ and (2)

$$
T O F=x_{1}+x_{2}-T_{\text {sum }}=y_{1}+y_{2}-T_{\text {sum }} .
$$

With (5) and (6) it is possible to recover position and TOF if at least one signal from each layer and only one more of the remaining values (out of $x_{1}, x_{2}, y_{1}, y_{2}$, and $t$ ) are available. It is to note that the position resolution for such a "recovered" event depends on the accuracy of the time sum information. So as the precision of $T_{\text {sum }}$ is usually worse than of $\sigma_{\mathrm{dx}}$ it is then the width $\sigma\left(T_{\text {sum }}\right)$ of the $T_{\text {sum }}$ peak determining the position precision of the recovered coordinate according to (4). Also the TOF resolution will degrade to $\sigma\left(T_{\text {sum }}\right)$ if $t$ is not available. However, if this decreased (worse) resolution is not seriously affecting the relevance of the acquired data, the redundancies of (5) and (6) can be used for event recovery when individual signals are "lost." This can be due to false threshold settings in the CFD and in case of multihit events when the time distance between particles is of the order of the electronic dead time. As will be shown in the next section the multihit ability of the delay-line anode is a consequence of this redundancy.

\section{Multihit Detection With the Helical Wire Anode}

The first condition to record a multihit event is the use of a multistop time-to-digital converter (TDC) with at least five channels, sufficient range and also sufficiently small timing bin size (time resolution). Analog TDCs (operating similar to an ADC) can have a very good time resolution but cannot handle multihits as defined in the introduction since the electronic circuits have a too long dead time.

Multistop TDCs use a different technique. Very fast clocks allow the time determination between two signals just by counting the clock pulses in this time window. No analog circuit is required, thus, the range is virtually unlimited and so is the number of hits to be acquired. Also, the double-hit dead-time can be very low. Using a Vernier method the time precision can be much shorter than the distance between two clock pulses. It is nowadays possible to achieve a bin size of 100 ps and below with such a method, yielding sufficient resolution for the absolute TOF timing and a decent position resolution. It is to note, however, that the time resolution of the front-end electronics (amplifiers and CFD) is significantly better than the bin size of today's multistop TDCs. ${ }^{1}$ This limits the position resolution of the delay-line anode. It is also the multihit dead-time of these TDCs that is determining the total electronic dead-time $\Delta t_{e}$. Typically $\Delta t_{e}$ is $10 \mathrm{~ns}$.

Equipped with such TDCs one can determine the position and time of certain multihit events with a HDL [21]. As long as the time distance $\Delta t_{\mathrm{pp}}$ between those two particles in a shower which are closest in time is bigger than the maximum dwell time of a signal on the delay-line $\left(=T_{\text {sum }}\right)$, there is no need for a special event recovery routine. Independently of the particle positions all five signals $x_{1}, x_{2}, y_{1}, y_{2}$, and $t$ from an "early" particle 1 arrive earlier than those of "late" particle 2 .

If $T_{\text {sum }}>\Delta t_{\mathrm{pp}}>\Delta t_{e}$, a time sum check is required to verify whether the first signal on a delay-line anode end belongs to the early or the late particle. Equations (5) and (6) might be needed to recover events because some of signals $x_{1}, x_{2}, y_{1}, y_{2}$ for the two particles can arrive within the electronic dead time on some delay-line end. However, at least on one of the delay-line ends of both the $X$ and $Y$ layer signals from both particles will be available, together with the corresponding MCP signals of both particles. The event can always be reconstructed if (5) and (6) are used for each particle's signals.

But for $\Delta t_{e}>\Delta t_{\mathrm{pp}}$ an event can only be reconstructed if the relative particle positions are separated by at least $\left(\Delta t_{e}-\right.$ $\left.\Delta t_{\mathrm{pp}}\right) \cdot v_{\perp}$ in $X$ and $Y$ coordinate. A detailed analysis can be found in [25]. This results in a cross-shaped "dead" region in a relative position plot (see Fig. in next section) and, thus, to a position dependent multihit efficiency for particles that arrive within the electronic dead time. Furthermore, if particles arrive

\footnotetext{
${ }^{1}$ The time precision of multistop TDCs is expected to soon reach $30 \mathrm{psec}$ [26]. If the TDC bin size $\Delta t$ dominates the time resolution ( $d x$ small) the position bin size $\Delta X$ is given by $\Delta t / 2 \cdot v_{\perp}$.
} 


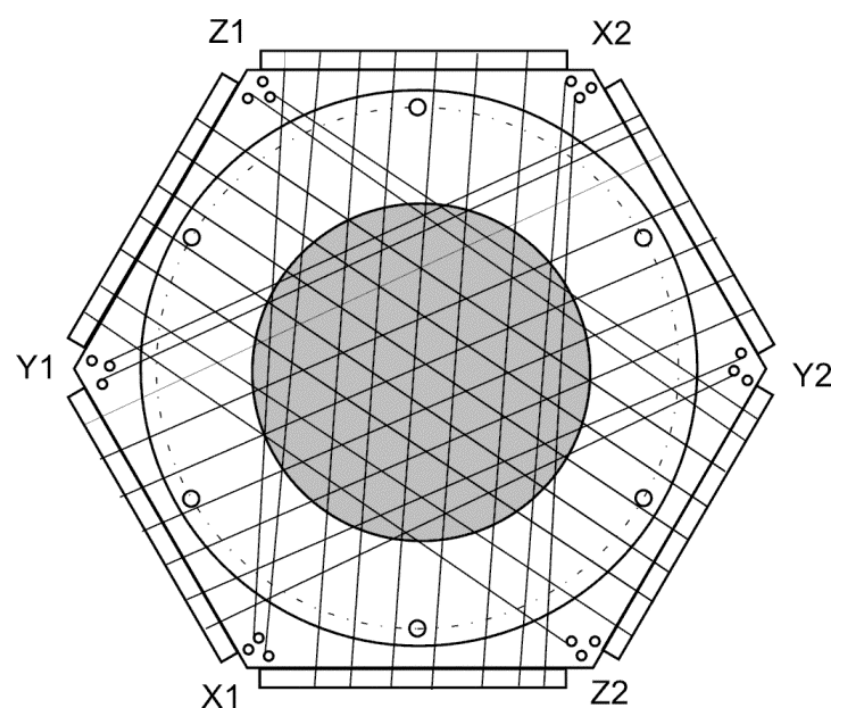

Fig. 2. Sketch of the Hexanode.

simultaneously, i.e., within the time sum peak width, the $X$ and $Y$ coordinates of the particles are ambiguous and only their distance can be determined. Note that these considerations are also valid for all other delay-line types that have only two delay lines for the two spatial coordinates.

As this situation is unsatisfactory for some applications we have developed a new anode for improved multihit performance, the so-called Hexanode.

\section{HEXANODE FOR IMPROVED MulTiHIT REAdOUT}

By using a third helical anode layer it is possible to resolve the ambiguity for simultaneously arriving particles [18]. Furthermore, the cross-shaped dead region collapses to a much smaller point-like shape of diameter $2 \Delta t_{e} \cdot v_{\perp}$ or smaller as will be shown later.

Fig. 2 shows a schematic drawing of the Hexanode. The perpendicular " $x$ " and " $y$ " layers of the HDL are now at a relative angle of $60^{\circ}$ and a third " $z$ " layer is introduced, again with 1 -mm distance and $60^{\circ}$ orientation relative to the other layers. Also, with three layers it is surprisingly easy to distribute the charge cloud from the MCP equally on all three layers and to obtain signals that allow a two-dimensional position determination with any two of the layers. The equations and consideration of the second section are valid here, too, just supplemented by an additional set for the $z_{1}$ and $z_{2}$ timing signals. Thus, one can define the hexagonal coordinates

$$
\begin{aligned}
u & =\left(x_{1}-x_{2}\right) \cdot v_{\perp} \\
v & =\left(y_{1}-y_{2}\right) \cdot v_{\perp} \\
w & =\left(z_{1}-z_{2}\right) \cdot v_{\perp}+o .
\end{aligned}
$$

$o$ is an offset parameter that guarantees the three layers having a common center, i.e., $u, v$, and $w$ are 0 in the center of the detector. One can then calculate the Cartesian position coordinates from any two of the hexagonal coordinates. If it is assumed that $u$ is aligned with $X$ it follows:

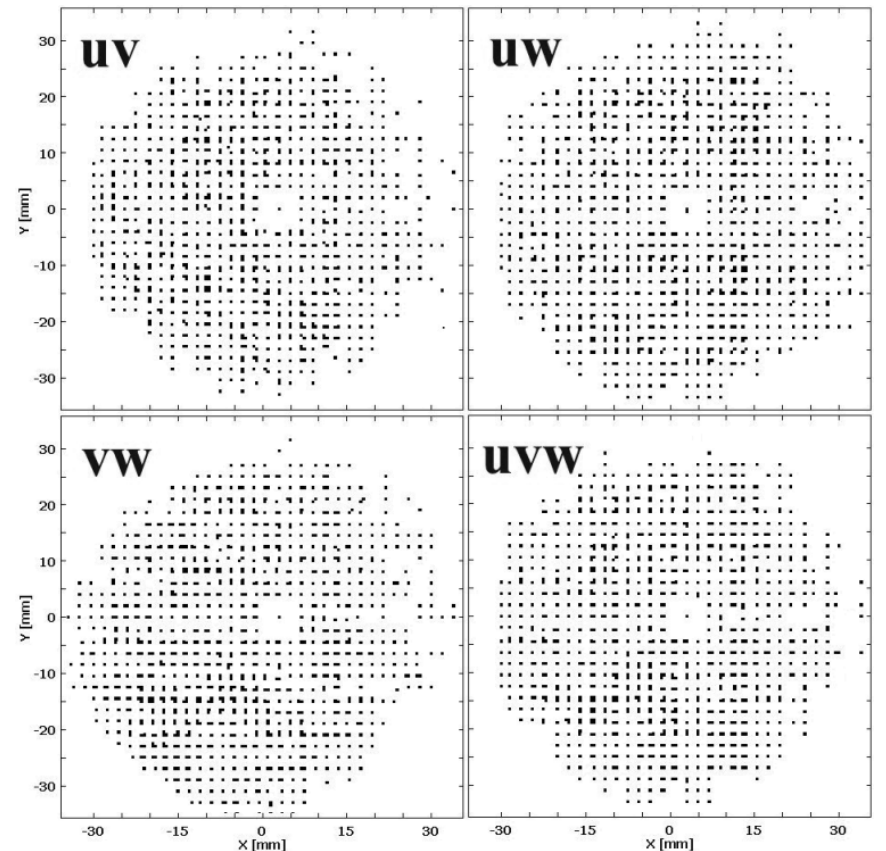

Fig. 3. Images of test mask obtained from the three independent hexagonal coordinate combinations and from a subset where all three coordinates are used (see text).

$$
\begin{aligned}
X u v & =u \\
Y u v & =\frac{1}{\sqrt{3}} \cdot(u-2 v) \\
X u w & =u \\
Y u w & =-\frac{1}{\sqrt{3}} \cdot(u+2 w) \\
X v w & =(v-w) \\
Y v w & =-\frac{1}{\sqrt{3}} \cdot(v+w) .
\end{aligned}
$$

Fig. 3 shows four representations of a test mask image (1-mm holes with 2-mm distance), obtained from the three hexagonal coordinate pair choices according to (8) and the corresponding image when all three layers are used $(X u / Y v w)$. A precise calibration is required and also a good linearity of the three delay-line layers is important. For the test anodes we found that the linearity is better than $0.3 \mathrm{~mm}$ over $70 \mathrm{~mm}$ even without additional software correction.

As $T_{\text {sum }}=z_{1}+z_{2}$ (3), (5), and (6) can be completed to give a redundant equation set for the Hexanode that can be used for event recovery. The additional coordinate set resolves the ambiguity for truly simultaneous particle hits. It also allows reducing the "dead" region: If the two particles hit with a distance larger than

$$
D_{\min }=2 v_{\perp} \cdot\left(\Delta t_{e}+\Delta t_{\mathrm{pp}}\right)
$$

there are sufficient coordinates to determine TOF and position of the two hits, even in a redundant way to allow cross checks. ${ }^{2}$ Fig. 4 shows the dead regions for the HDL and the Hexanode in comparison. Note again, that these considerations are also valid for more than two incoming particles. Assuming perfect

\footnotetext{
${ }^{2} \mathrm{~A}$ more detailed analysis reveals that the "dead" region has the shape of a regular David star that fits into a circle with $2 v_{\perp} \cdot\left(\Delta t_{e}+\Delta t_{\mathrm{pp}}\right)$ diameter.
} 

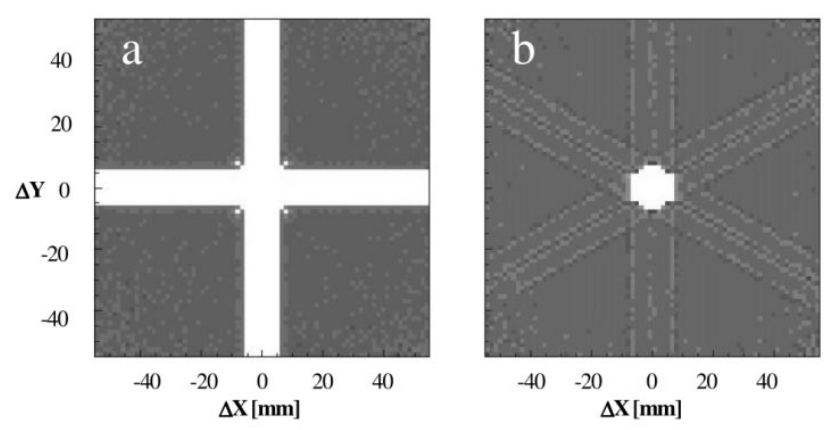

Fig. 4. Monte Carlo simulation of the "dead" regions for two particles that arrive with relative positions $\Delta X, \Delta Y$ on a standard HDL (a) and on a Hexanode (b) of $80-\mathrm{mm}$ active diameter. The relative arrival time of the particles in this simulation is $2.5 \mathrm{~ns}$ for the HDL and $0 \mathrm{~ns}$ for the Hexanode. Only in the dark regions, the position and time of both particles can be reconstructed.

signal recovery, positions of up to four simultaneous particles, i.e arriving within $\sigma\left(T_{\text {sum }}\right)$, could theoretically be reconstructed unambiguously (also assuming zero dead time). Even more simultaneous particles could be registered unless these are not arranged in rather peculiar (and, thus, unlikely) triangular patterns. However, for nonzero dead time the situation is more complicated. A third particle can interfere with signals of two earlier particles and, thus, even reduce the number of usable signals for position reconstruction of the two earlier particles. This situation is currently analyzed in more detail.

Still the Hexanode has a considerably improved ability to resolve multihits compared to the HDL. While the HDL responds to multihits adequately as long as $\Delta t_{\mathrm{pp}}>\Delta t_{e}$, the third layer of the Hexanode allows an extension beyond the electronic dead time limit. The MCP signals $(t)$ of the particles are not used in such case. Position and time can be reconstructed from the signals on the three layers as long as the particles' distance is larger than $D_{\min }$, or in less precise words: if the particles do not arrive at the same time and at the same position.

This situation is similar for pixel type anodes. So the Hexanode matches the multihit performance of a pixel anode with pixel size $D_{\min }$, but it uses much less electronic circuits (only six or seven) and the position resolution is much superior to any pixel arrangement. Of course, for those events with $\Delta t_{\mathrm{pp}}>\Delta t_{e}$ the position and time resolution is considerably better.

\section{First Multihit ExPeriments Using the HeXanode}

It is rather simple to verify the imaging performance and linearity of the Hexanode, also it is straightforward to prove the redundant imaging between layer pairs. But it is quite an undertaking to set up a multihit test apparatus. A pulsed UV laser and optics to produce double (photon) hits in a controlled way will be set up in the future. So far we use data from experiments, where highly differential scattering cross sections are obtained with a Hexanode in multihit operation and compare the results with expectations. Experiments have shown that the method indeed works well [27], [28].

In a "real" experiment there are additional obstacles that challenge the application of the above considerations. Signals might
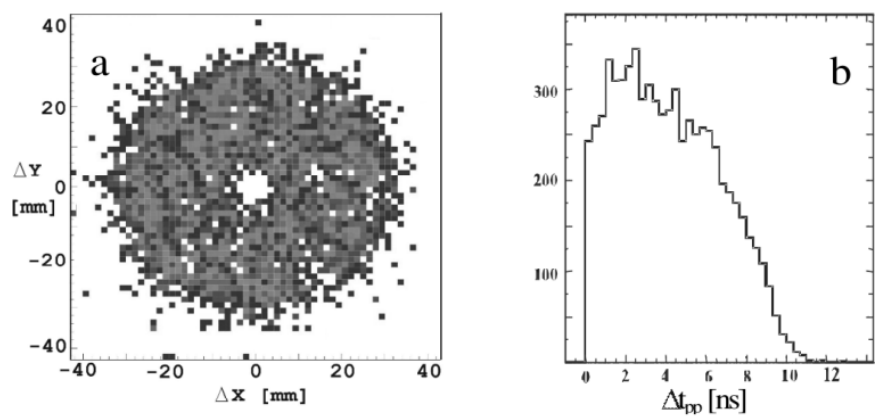

Fig. 5. Relative position (a) and relative time difference plots (b) for the two electrons from $D_{2}$ after photo-dissociation (see text). The electrons are scattered over 35-mm relative distance and arrive both within a $11 \mathrm{~ns}$ time window on the Hexanode detector. Note that for all events investigated here $\Delta t_{\mathrm{pp}}$ is smaller than $\Delta t_{e}$. In the position plot only events with $\Delta t_{\mathrm{pp}}>4 \mathrm{~ns}$ are displayed.

be "lost" not only due to electronic dead time but also because of nonperfect threshold settings. Furthermore, reflections after the "real" signals or noise can result in additional signals that enter the time sequences. These problems could be resolved by using a rather complicated (but straightforward) event reconstruction algorithm. Due to the redundancies that can be used to verify a reconstructed event, it is possible to sort out false signals and allow event reconstruction from pulse sequences with "false" lost signals without producing erroneous reconstructions. Also only a small percentage of real events are obscured and lost so that the differential cross sections are not significantly affected.

The most "demanding" experiment in terms of multihit capability so far has been performed at the advanced light source (ALS) in Berkeley, CA [27]. A photon beam of 79-eV photon energy intersected a molecular gas jet target of $D_{2}$ in a COLTRIMS reaction microscope [14]. Absorption of a photon leads with a few percent probability to a complete fragmentation of the molecule into two positively charged $D^{+}$ ions and two electrons. A combination of electrostatic and magnetic solenoid fields guides the charged fragments to two position-sensitive $80 \mathrm{~mm} \mathrm{MCP}$ detectors facing each other. The geometrical solid angle of this spectrometer is $4 \pi$ for both electrons and ions. From the TOF and impact position of each fragment its final state momentum in all three spatial dimensions can be determined. The ion-side MCP was equipped with a HDL while the electron hits have been registered with a Hexanode. Although ions and electrons share about the same kinetic energy, the electrons are much faster due to their lower mass and arrive within a 10-ns time window relative to each other on the MCP.

Fig. 5 shows a plot of the electrons' relative position distribution $(\Delta X, \Delta Y)$ and the relative time difference distribution $\Delta t_{\mathrm{pp}}$, as obtained after event reconstruction. The ions arrive with a typical time difference of a few hundred nanoseconds so that a HDL had sufficient multihit capability to detect the pair. The electronic dead time $\Delta t_{e}$ for both detectors was about $12 \mathrm{~ns}$.

Fig. 6 shows cross sections for the polar angle distribution of one electron when the other electron has a fixed polar angle with reference to the polarization vector $\epsilon$ of the incoming synchrotron light, integrated over all azimuth angles. The cross sections for equal energy sharing of the electrons show the typical 


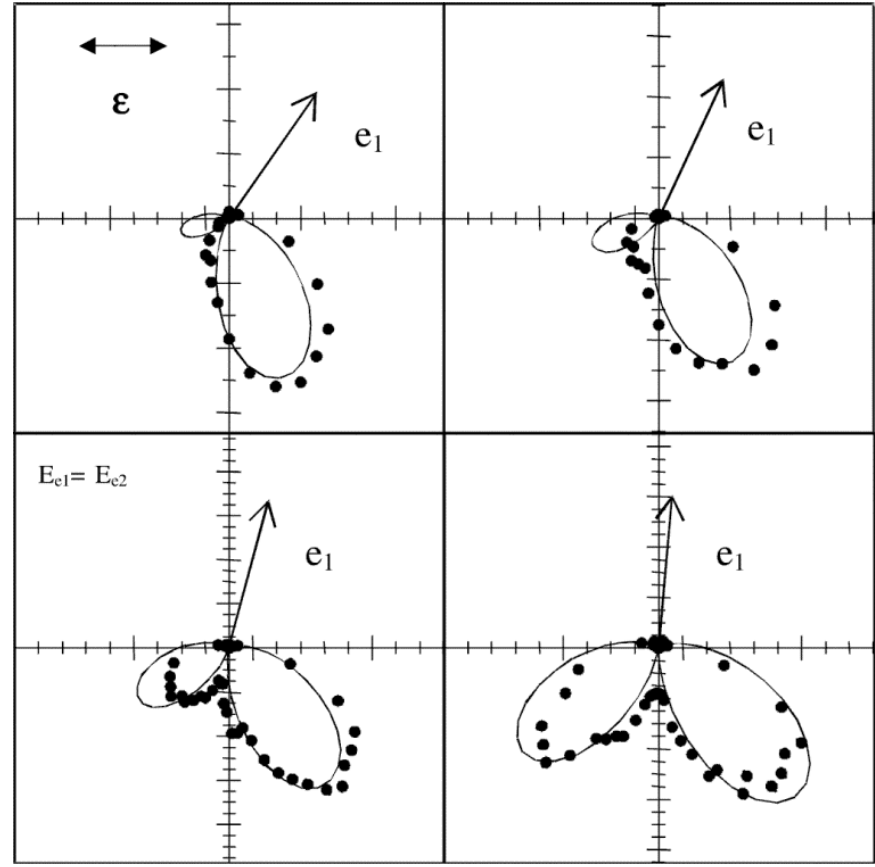

Fig. 6. Cross section of relative polar angle of the two electrons after photo-dissociation with respect to the polarization vector $\epsilon$ of the synchrotron light for equal energy sharing between the electrons. The shape of the cross sections are in fair agreement with theory (see text)

lobes and nodes as expected for such experiments. The results are in fair agreement with theoretical expectations (solid lines) [29].

These and other experiments provide solid evidence that the Hexanode works as expected. These investigations have been performed under real experimental conditions, which are usually less "forgiving" and less carefully prepared than in a test environment.

\section{SUMMARY AND OUTLOOK}

We have shown that the Hexanode can significantly expand the application of delay-line anodes for experiments with serious multihit demands. The Hexanode can resolve multihit events as long as any two particles of a shower are not arriving at the same time and at the same position, with limits given by the electronic dead-time and anode size.

Such multihit ability is combined with high and uniform position resolution that cannot be achieved with pixel-type anodes. The electronic expenditure is comparably low.

It should be mentioned that with the three layer arrangement it is also possible to build a delay-line anode with central hole, e.g., to allow a beam pass through.

In order to maximize the multihit ability of the Hexanode it is desirable to minimize the electronic dead-time $\Delta t_{e}$ and, thus, $D_{\min }$. For $\Delta t_{e}=10 \mathrm{~ns}$ and simultaneously arriving particles $\left(\Delta t_{\mathrm{pp}}=0\right)$, the minimum particle distance $D_{\min }$ is typically smaller than $10 \mathrm{~mm}$ for a Hexanode with $80 \mathrm{~mm}$ active diameter.

A new generation of amplifiers that will be used in the next experiments have a band width of $6 \mathrm{GHz}$ and are, thus, not broadening the signals from the delay-line or the MCP. The FWHM of signals from the MCP $(t)$ is found to be $2.5 \mathrm{~ns}$. The signals from the delay-line have between 4 and $5 \mathrm{~ns}$ FWHM due to the size of the charge cloud foot print on the layers.

The fastest commercial CFDs have $5 \mathrm{~ns}$ dead time and some novel TDC circuits have also pulse-pair dead-times of $5 \mathrm{~ns}$ or less. So we expect to reduce the dead time by at least a factor of two compared to the present situation.

As the next step we are considering to introduce fast flash-ADCs ( $>1 \mathrm{GHz}$ sampling rate) to read out the amplified signals' shapes and perform an on-board shape (timely position) analysis using FPGA circuits. Thus, it should be possible to determine the timing of the signals without CFD or TDC and to reach the dead time limit given by the FWHM of the detector signals. For the MCP signal this should result in a dead time as low as $2.5 \mathrm{~ns}$.

We expect that the Hexanode's combination of certain multihit ability and high position resolution at comparably low system costs will be a valuable tool for many new applications.

\section{REFERENCES}

[1] G. W. Fraser, "X- and $\gamma$-ray imaging using microchannel plates," Nucl. Instrum. Methods Phys. Res. A, Accel. Spectrom. Detect. Assoc. Equip., vol. 221, pp. 115-130, 1984.

[2] M. Lampton and F. Paresce, "The Ranicon: A resisitive anode image converter,” Rev. Sci. Instrum., vol. 45, pp. 1098-1105, 1974.

[3] C. Martin, P. Jelinsky, M. Lampton, R. F. Malina, and H. O. Anger, "Wedge-and-strip anodes for centroid-finding position-sensitive photon and particle detectors," Rev. Sci. Instrum., vol. 52, pp. 1067-1074, 1981.

[4] J. S. Lapington and B. Sanderson, "Microchannel plate limited resolution with the Vernier anode," in Proc. SPIE, vol. 4139, 2000, pp. 242-249.

[5] J. S. Morgan, D. C. Slater, and J. G. Timothy, "Centroid position measurements and subpixel sensitivity variations with the MAMA detector," Appl. Opt., vol. 28, no. 6, pp. 1178-1192, 1989.

[6] E. Kellogg, P. Henry, S. Murray, L. Van Spreybroeck, and P. Bjorkholm, "High resolution imaging x,ray detector," Rev. Sci. Instrum., vol. 47, no. 3, pp. 282-290, 1976.

[7] O. H. W. Siegmund, A. S. Tremsin, J. V. Vallergas, and J. Hull, "Cross strip anodes for microchannel plate detectors," IEEE Trans. Nucl. Sci., vol. 48, pp. 430-434, June 2001.

[8] S. E. Sobottka and M. B. Williams, "Delay line readout of microchannel plates," IEEE Trans. Nucl. Sci., vol. 35, pp. 348-351, Feb. 1988.

[9] P. G. Friedman, R. A. Cuza, J. R. Fleischman, C. Martin, D. Schiminovich, and D. J. Doyle, "Multilayer anode with crossed serpentine delay lines for high spatial resolution readout of microchannel plate detectors," Rev. Sci. Instrum., vol. 67, no. 2, pp. 596-608, 1996.

[10] H. Keller, G. Klingelhöfer, and E. Kankeleit, "A position sensitive microchannelplate detector using a delay line readout anode," Nucl. Instrum. Methods Phys. Res. A, Accel. Spectrom. Detect. Assoc. Equip., vol. A258, pp. 221-224, 1987.

[11] O. H. W. Siegmund, P. Jelinsky, S. Jelinskyk, J. Stock, J. Hull, D. Doliber, J. Zaninovich, A. S. Tremsin, and K. Kromer, "High resolution cross delay line detectors for the GALEX mission," in Proc. SPIE, vol. 3765, 1999, pp. 429-440.

[12] J. H. D. Eland, "Simple two-dimensional position-sensitive detector with short dead-time for coincidence experiments," Meas. Sci. Technol., vol. 5, pp. 1501-1504, 1994.

[13] O. Jagutzki, J. Barnstedt, U. Spillmann, L. Spielberger, V. Mergel, K. Ullmann-Pfleger, M. Grewing, and H. Schmidt-Böcking, "Fast-position and time-sensitive readout of image intensifiers for single-photon detection," in Proc. SPIE, vol. 3764, 1999, pp. 61-69.

[14] R. Dörner, V. Mergel, O. Jagutzki, L. Spielberger, J. Ullrich, R. Moshammer, and H. Schmidt-Böcking, "Cold target recoil ion momentum spectroscopy: A 'momentum microscope' to view atomic collision dynamics," Phys. Rep., vol. 330, no. 2-3, pp. 95-192, June 2000

[15] J. Becker, K. Beckord, U. Werner, and H. O. Lutz, "A system for correlated fragment detection in dissociation experiments," Nucl. Instrum. Methods Phys. Res. A, Accel. Spectrom. Detect. Assoc. Equip., vol. A337, pp. 409-415, 1994. 
[16] S. Martin, L. Chen, A. Denis, R. Bredy, J. Bernard, and J. Desesquelles, "Excitation and fragmentation of $\mathrm{C}_{60}^{r+}(r=3-9)$ in $\mathrm{Xe}^{30+}{ }_{-} \mathrm{C}_{60}$ collisions," J. Phys. A, Math. Gen., vol. A62, pp. 022 707/1-5, 2000.

[17] A. Cerezo, T. J. Godfrey, S. J. Sijbrandij, G. D. W. Smith, and P. J. Warren, "Performance of an energy-compensated three-dimensional atom probe," Rev. Sci. Instrum., vol. 69, pp. 49-58, 1998.

[18] D. Kella, M. Algranati, H. Feldman, O. Heber, H. Kovner, E. Malkin, E. Miklazky, R. Naaman, D. Zajfman, J. Zajfman, and Z. Vager, "A system for Coulomb explosion imaging of small molecules at the Weizmann institute," Nucl. Instrum. Methods Phys. Res. A, Accel. Spectrom. Detect. Assoc. Equip., vol. A329, pp. 440-452, 1993.

[19] O. Jagutzki, V. Mergel, K. Ullmann-Pfleger, L. Spielberger, U. Meyer, R. Dörner, and H. Schmidt-Böcking, "Fast position and time resolved readout of micro-channelplates with the delay-line technique for single particle and photon detection," in Proc. SPIE, vol. 3438, 1998, pp. 322-334.

[20] O. Jagutzki, H. Schmidt-Böcking, V. Mergel, A. Cerezo, and M. Huang, "Device and Method for Two-Dimensional Detection of Particles," U.K. Patent 0003 261.5, Aug. 15, 2001.

[21] W. Koenig, A. Faibis, E. P. Kanter, Z. Vager, and B. J. Zabransky, "A multiparticle 3D imaging technique to study the structure of molecular ions," Nucl. Instrum. Methods Phys. Res. B, Beam Interact. Mater. At., vol. B10, pp. 259-265, 1985.
[22] A. Faibis, W. Koenig, E. P. Kanter, and Z. Vager, "MUPPATS - A multiparticle 3D imaging detector system," Nucl. Instrum. Methods Phys. Res. B, Beam Interact. Mater. At., vol. B13, pp. 673-677, 1986.

[23] A. Belkacem, A. Faibis, E. P. Kanter, W. Koenig, R. E. Mitchell, Z. Vager, and B. J. Zabransky, "A new type of multiparticle imaging detector," Rev. Sci. Instrum., vol. 61, pp. 945-952, 1990.

[24] R. A. Lewis, N. S. Fore, C. Hall, W. Helsby, A. Jones, B. Parker, I. Sumner, and J. S. Worgan, "A highly parallel imaging gas counter for synchrotron radiation diffraction," Nucl. Instrum. Methods Phys. Res. A, Accel. Spectrom. Detect. Assoc. Equip., vol. A348, pp. 237-240, 1994.

[25] O. Jagutzki, V. Mergel, K. Ullmann-Pfleger, L. Spielberger, U. Spillmann, R. Dörner, and H. Schmidt-Böcking, "A broad-application microchannel-plate detector system for advanced particle or photon detection tasks: Large area imaging, precise multihit timing information and high detection rate," Nucl. Instrum. Methods Phys. Res. A, Accel. Spectrom. Detect. Assoc. Equip., vol. A477, pp. 244-249, 2002.

[26] A. Larsch, personal communication, acam-GmbH, Karlsruhe, Germany, 2001.

[27] T. Weber, Ph.D. dissertation, Universität Frankfurt, Frankfurt, Germany.

[28] N. Saito, Personal Communication, National Institute of Advanced Industrial Science and Technology, Ibaraki, Japan, 2001.

[29] M. Walter and J. S. Briggs, "Photo-double ionization of molecular hydrogen," J. Phys. B, At. Mol. Opt. Phys., vol. B32, pp. 2487-2507, 1999. 